\title{
Metabolic pathway for degradation of 2-chloro-4- aminophenol by Arthrobacter sp. SPG
}

\author{
Pankaj Kumar Arora ${ }^{1 *}$, Tapan Kumar Mohanta ${ }^{1}$, Alok Srivastava ${ }^{2}$, Hanhong Bae ${ }^{1 *}$ and Vijay Pal Singh ${ }^{2^{*}}$
}

\begin{abstract}
A degradation pathway of 2-chloro-4-aminophenol (2C4AP) was studied in an Arthrobacter sp. SPG that utilized 2C4AP as its sole source of carbon and energy. The 2C4AP degradation was initiated by a 2C4AP-deaminase that catalyzed the conversion of $2 \mathrm{C} 4 \mathrm{AP}$ into chlorohydroquinone $(\mathrm{CHQ})$ with removal of ammonium ion. In the next step, a CHQ-dehalogenase dehalogenated CHQ to hydroquinone (HQ) that cleaved into $\gamma$-hydroxymuconic semialdehyde by a HQ-dioxygenase. The 2C4AP degradation was also investigated in sterile and non-sterile soil microcosms using strain SPG. The results show that the SPG cells degraded 2C4AP more rapidly in sterile soil than non-sterile soil. Our studies showed that strain SPG may be used for bioremediation of 2C4AP-contaminated sites. This is the first report of the 2C4AP degradation by any bacteria.
\end{abstract}

\section{Introduction}

Chloroaminophenols (CAPs) including 2-chloro-4-aminophenol (2C4AP) and 4-chloro-2-aminophenol (4C2AP) are widely used as hair dyes and have been classified as toxic substances because of their carcinogenicity [1]. CAPs have been released into soil and water as the by-products during the synthesis of cosmetic dyes and chemicals. They may also release into soil because of microbial degradation of various chemicals including 3 -chloronitrobenzene and 4-chloro-2-nitrophenol (4C2NP) [2,3].

Few bacteria capable of utilizing CAPs as their sole sources of carbon and energy have been identified and characterized [1,4]. Examples are Exiguobacterium sp. PMA [4] and Burkholderia sp. RKJ 800 [1]. Both of the bacterial strains utilized 4C2AP as the sole source of carbon and energy. The complete mineralization of 4C2AP was studied in Burkholderia sp. RKJ 800 that degraded it with release of ammonium and chloride ions [1]. The 4C2AP degradation was initiated with the formation of 4-chlorocatechol that was further degraded via ring cleavage [1].

The bacterial degradation of CAPs may be initiated by one of the following mechanisms: (i) the removal of

\footnotetext{
*Correspondence: arora484@gmail.com; hanhongbae@ynu.ac.kr; singhvp03@rediffmail.com

${ }^{1}$ School of Biotechnology, Yeungnam University, Gyeongsan 712-749, Republic of Korea

${ }^{2}$ Department of Plant Science, Faculty of Applied Sciences, MJP Rohilkhand University, Bareilly 243006, India
}

ammonium ion from a CAP by a deaminase [1]; (ii) the ring cleavage of a CAP by an aminophenol dioxygenase [5]; (ii) the dehalogenation of a CAP by a dehalogenase [4]; and (iii) the acetylation of a CAP [2,3,6].

In this communication, we have reported degradation of 2C4AP by Arthrobacter sp. SPG. Strain SPG was previously isolated from the soil collected from a pesticide contaminated site, India by an enrichment method using 4-nitrophenol [7]. Strain SPG utilized 4-nitrophenol, 2chloro-4-nitrophenol (2C4NP), 2-nitrobenzoate, 3-methyl4-nitrophenol and nitrocatechol as its sole sources of carbon and energy [7]. In addition, Arthrobacter sp. SPG is also capable of utilizing 2C4AP as its sole source of carbon and energy [8]. The aim of this work is to study the degradation pathway of 2C4AP by Arthrobacter sp. strain SPG and monitor the 2C4AP degradation potential of the same strain in soil under laboratory conditions.

\section{Results}

Growth and degradation studies

Arthrobacter sp. SPG uses $0.3 \mathrm{mM} \mathrm{2C4AP}$ as its sole source of carbon and energy and mineralized 2C4AP completely within $48 \mathrm{~h}$ (Figure 1a). The growth of the cells of strain SPG initiated after the incubation of $12 \mathrm{~h}$ and the maximum absorbance at $600 \mathrm{~nm}$ reached was 0.15 within 48 hours of incubation. The cells of strain SPG released stoichiometric amounts of chloride and ammonium ions during the degradation of 2C4AP. The time course analysis of ammonia and chloride releases 


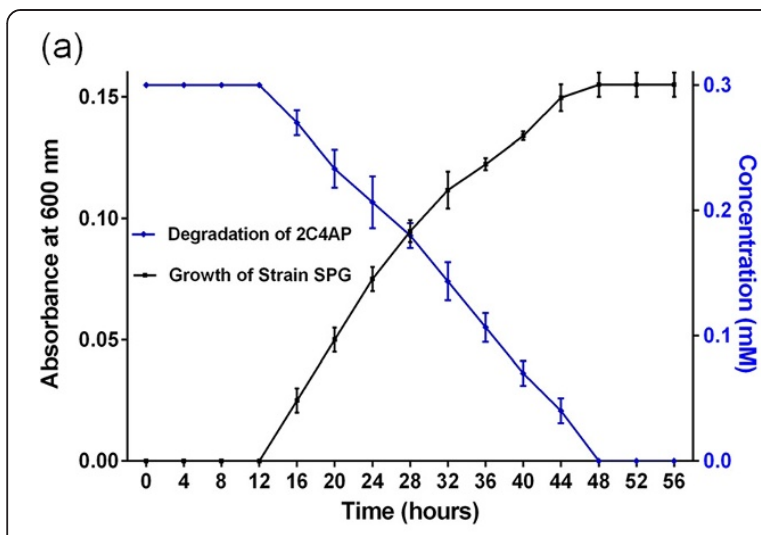

(b)

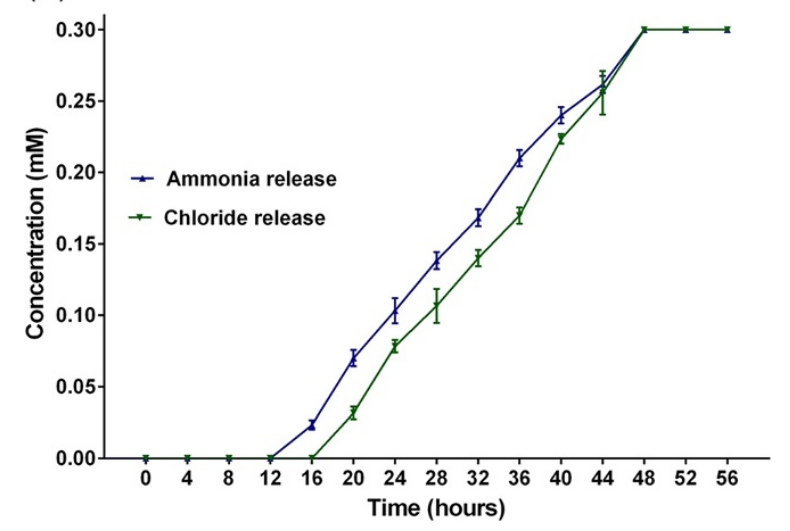

Figure 1 Growth and degradation studies. (a) Utilization and degradation of 2-chloro-4-aminophenol by Arthrobacter sp. SPG, and (b) Estimation of ammonium and chloride ions during the degradation of 2-chloro-4-aminophenol by Arthrobacter sp. SPG.

showed that the initiation of the ammonia release occurred before the initiation of the chloride release. These data suggest that the 2C4AP degradation was initiated with ammonia release and followed by chloride release (Figure 1b).

\section{Identification of metabolites}

High performance liquid chromatography analysis of the samples collected at different intervals showed complete disappearance of the peak of 2C4AP within $48 \mathrm{~h}$ (Figure 2). In the samples of 0 and $12 \mathrm{~h}$, a single peak of $2 \mathrm{C} 4 \mathrm{AP}$ was detected with the retention time of $13.36 \mathrm{~min}$. However, in the sample of $24 \mathrm{~h}$, a peak with retention time $6.89 \mathrm{~min}$ (referred to as metabolite 1) appeared along with the peak of the 2C4AP. In the sample of $36 \mathrm{~h}$, the peak of metabolite 11 with the retention time of $5.09 \mathrm{~min}$ was detected along with the peaks of metabolite 1 and the 2C4AP. In the sample of $48 \mathrm{~h}$, the peaks of both metabolites and the 2C4AP have disappeared suggesting the complete degradation of 2C4AP by Arthrobacter sp. SPG. The retention time of metabolite I and II were matched with authentic

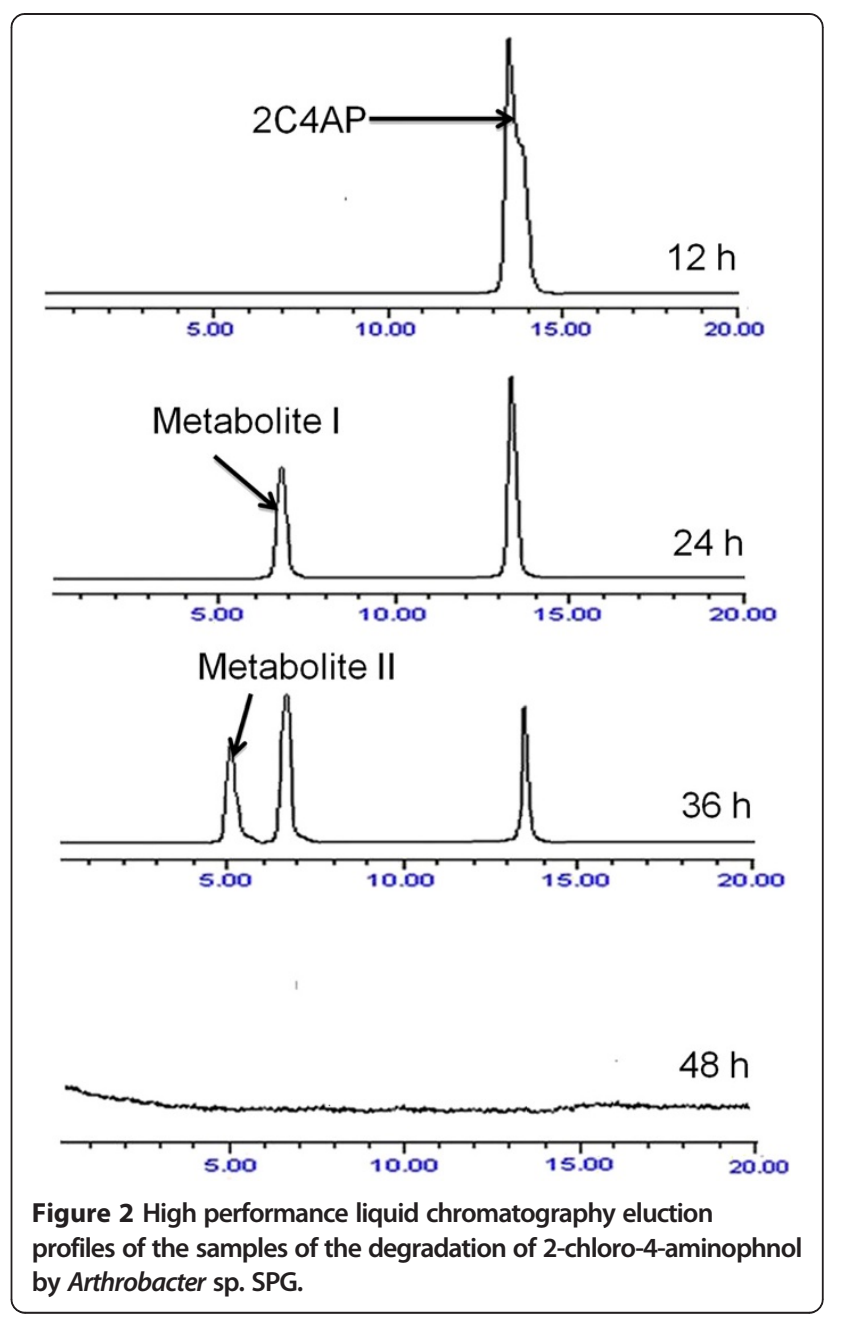

chlorohydroquinone (CHQ) and hydroquinone (HQ), respectively.

To identify both metabolites, samples ( 24 and 36 h) were analyzed with gas chromatography-mass spectrometry (GC-MS). The results of GC-MS analysis showed that the molecular ions of the metabolite I and II were observed at $m / z 144$ and $m / z 110$, respectively. The mass fragment patterns of metabolite I and II were equivalent with that of authentic standards of CHQ and HQ. On the basis of analytical analysis of the samples by HPLC and GC-MS, metabolite I and II were identified as CHQ and $H Q$, respectively.

\section{Enzyme assays}

We have detected enzyme activities of a 2C4APdeaminase, a CHQ-dehalogenase and a HQ-dioxygenase in the crude extracts of 2C4AP induced cells of strain SPG. The 2C4AP-deaminase activity showed the stoichiometric release of ammonium ions and the resulting product was identified as CHQ by the GC-MS. The CHQ-dehalogenase activity showed the stoichiometric 
release of chloride ions and the resulting product was detected as HQ by GC-MS. The HQ dioxygenase activity showed the disappearance of the peak of HQ at $289 \mathrm{~nm}$ with appearance of the peak of $\gamma$-hydroxymuconic semialdehyde, a ring-cleavage product of HQ at $320 \mathrm{~nm}$ suggesting the ring-cleavage of the HQ into $\gamma$-hydroxymuconic semialdehyde.

\section{Microcosm studies}

Microcosm studies showed that the cells of strain SPG were able to degrade 2C4AP in microcosms with sterile as well as non-sterile soil within 8 and 10 days, respectively (Figure 3). No degradation was found at initial 2 days in sterile soil whereas in test microcosm with non-sterile soil, no degradation was observed at initial 4 days. In the sterile soil, the degradation was $12 \%$ at 3 days and $30 \%$ at 4 days. After 6 days, the degradation rate was $65 \%$ in sterile soil whereas almost complete degradation was observed at 8 days in test microcosm with sterile soil. In the test microcosm with non-sterile soil, the degradation was $30 \%, 45 \%, 65 \%$, and $85 \%$ in 6 days, 7 days, 8 days and 9 days, respectively. The complete degradation of 2C4AP was observed at 10 days in test microcosm with sterile soil. In both of the control microcosms, with sterile and non-sterile soil, there was no degradation within 10 days. GC-MS analysis of the samples showed the presence of CHQ and HQ in the 6 days sample of sterile soil microcosm and the 7 days sample of the non-sterile soil microcosm. No intermediate was detected in any other sample. All microcosm experiments were performed under optimum conditions which include $\mathrm{pH} 7.0$, temperature $30^{\circ} \mathrm{C}, 100 \mathrm{ppm} 2 \mathrm{C} 4 \mathrm{AP}$ and the inoculum bacterial size $2 \times 10^{8} \mathrm{CFU} \mathrm{g}^{-1}$ soil.

\section{Discussion}

Arthrobacter sp. SPG utilized 2C4AP as its sole source of carbon and energy and degraded it with release of stoichiometric amounts of chloride and ammonium ions.

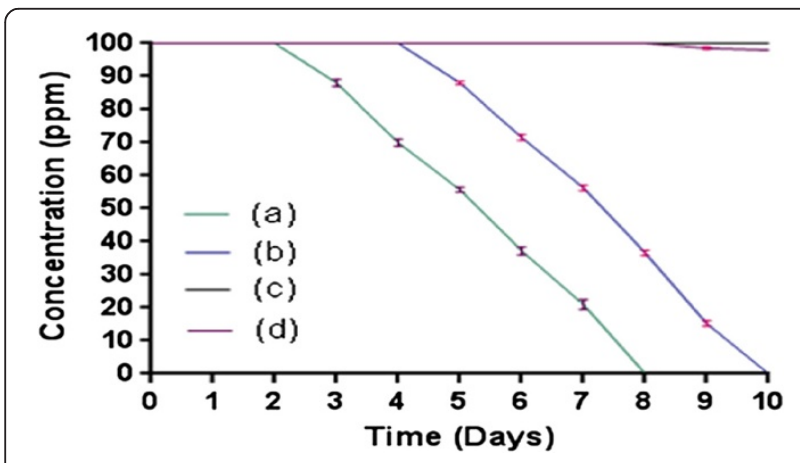

Figure 3 Degradation of 2C4AP by Arthrobacter sp. SPG in (a) microcosm with sterile soil, (b) microcosm with non-sterile soil, (c) Un-inoculated control with sterile soil and (d) Un-inoculated control with non-sterile soil.
The CHQ was detected as the initial metabolite of the degradation pathway of 2C4AP. The enzyme 2C4APdeaminase was responsible for the $\mathrm{CHQ}$ formation in the 2C4AP degradation. Literature studies showed that the CHQ is a common metabolite in the degradation pathway of several chlorinated compounds [9-13]. Reddy et al. [11] reported that the CHQ was degraded either via $\mathrm{HQ}$ or via 2-chlorotrihydroxybenzene in degradation pathway of 2,4,6-trichlorophenol in Phanerochaete chrysosporium. Miyauchi et al. [12,13] showed that CHQ cleaved to maleylacetate or dechlorinated to HQ in the degradation pathway of gamma-hexachlorocyclohexane in Sphingomonas paucimibilis UT260. Arthrobacter sp. SJCon degraded 2C4NP via a CHQ pathway in which CHQ was cleaved to maleylacetate by a CHQ-dioxygenase [14]. Burkholderia sp. RKJ 800 [9] and Rhodococcus imtechensis RKJ300 [10] degraded 2C4NP via a CHQ pathway in which CHQ dehalogenated to HQ. In this study, we have also detected HQ as a metabolite of degradation of 2C4AP by strain SPG. Furthermore, we have also detected the activity of the CHQ dehalogenase in the crude extract of the 2C4AP-induced cells of strain SPG that confirmed the formation of the HQ from CHQ with release of chloride ion. The HQ was also detected as a metabolite of degradation pathway of 4-nitrophenol in a various Gram-positive bacteria $[7,14,15]$. In the degradation pathway of 4-nitrophenol, HQ was cleaved into gammahydroxymuconic semialdehyde by a HQ-dioxygenase $[7,14]$. We have also detected the HQ-dioxygenase activity in the crude extracts of the 2C4AP-induced cells of strain SPG that suggested the cleavage of HQ into gammahydroxymuconic semialdehyde.

On the basis of the identified metabolites and the results of the enzyme assays, we have proposed the 2C4AP degradation pathway for Arthrobacter sp. SPG (Figure 4). The 2C4AP degradation was initiated with release of ammonium ion and the formation of CHQ that further dehalgenated to HQ with release of chloride ion. The further degradation of $\mathrm{HQ}$ was proceeded via ring cleavage.

The 2C4AP degradation pathway identified in Arthrobacter sp. SPG was compared with the degradation pathway of 4C2AP in Burkholderia sp. RKJ 800 [1]. It was observed that the initial mechanism of degradation of 2C4AP in strain SPG was similar with that of the 4C2AP degradation in strain RKJ 800 [1]. Both strains initiated the degradation of an isomer of CAPs with removal of ammonium ion by a deaminase [1]. The difference in the degradation pathways of $2 \mathrm{C} 4 \mathrm{AP}$ and $4 \mathrm{C} 2 \mathrm{AP}$ is that the chloride release was occurred before the ring cleavage in the degradation pathway of 2C4AP whereas in the degradation of $4 \mathrm{C} 2 \mathrm{AP}$, chloride was released after the ring cleavage [1].

The cells of strain SPG were capable of degrading 2C4AP in the soil under laboratory conditions. The rate 


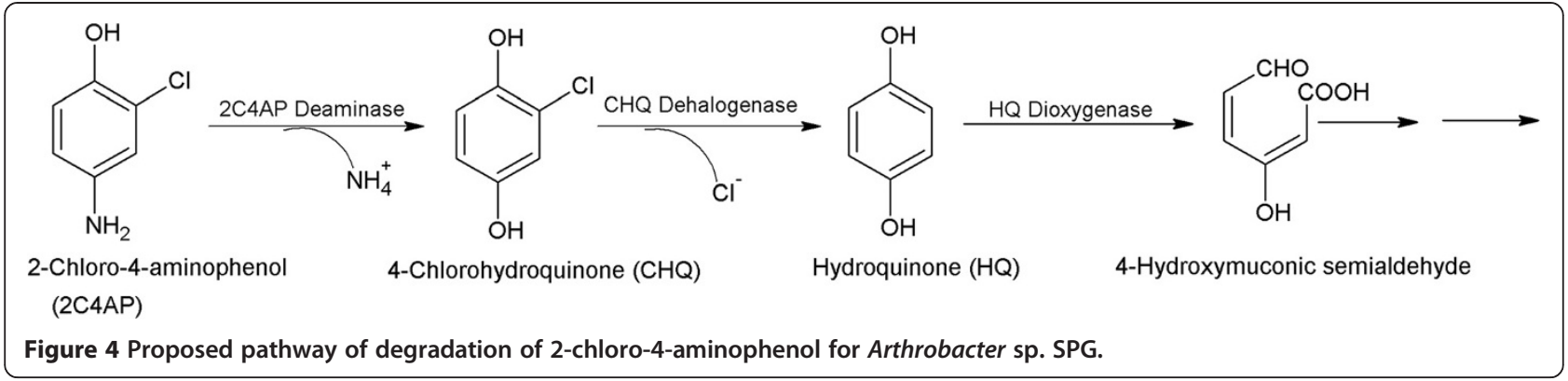

of the 2C4AP degradation was faster in sterile soil than non-sterile soil. The 2C4AP degradation pattern was very similar in sterile and non-sterile soil. The major difference is an extended lag phase of strain SPG in nonsterile soil due to the presence of natural microbiota. These data indicate that strain SPG initially compete with natural microbiota in non-sterile soil, then acclimated and degraded 2C4AP. Literature studies showed that two kinds of mechanisms may involve in microcosm degradation studies with non-sterile soils; (i) bacterial inhibition in which indigenous microorganisms produced antagonistic substances to inhibit the growth of an inoculated bacterium $[14,16]$ and (ii) microbial cooperation in which indigenous microorganisms enhanced the degradation rate by utilizing xenobiotics or their metabolites $[14,17]$. In our study, we have not observed the enhancement of degradation rate in non-sterile soil that indicated there is no role of indigenous microbiota to enhance the 2C4AP degradation.

Previous studies showed that several bacteria have ability to degrade various chlorophenols in microcosms with sterile and non-sterile soil $[1,4,9,18,19]$. Rhodococcus imtechensis RKJ300 degraded 2C4NP in soil and the degradation rate was faster in the non-sterile soil than sterile soil $[14,18]$. Similar findings have been observed in a 4C2NP-mineralizing bacterium, Exiguobacterium sp. PMA that degraded $4 \mathrm{C} 2 \mathrm{NP}$ with faster rate in nonsterile soil [4]. The equal rate of the degradation of 4C3NP in sterile and non-sterile soil was observed in Pseudomonas sp. JHN [19]. Another bacterium, Burkholderia sp. RKJ 800 degraded 4C2AP and 2C4NP in soil and the degradation rate of the both compounds was faster in non-sterile soil as compared to sterile soil $[1,9]$. In this study, strain SPG degraded 2C4AP more rapidly in sterile soil than non-sterile soil.

\section{Conclusions}

Arthrobacter sp. SPG utilized 2C4AP as its sole source of carbon and energy and degraded it via a novel pathway with the formation of CHQ and HQ as the metabolites. Initially, a 2C4AP deaminase catalyzed the novel conversion of 2C4AP to CHQ with release of the ammonium ion. In the next step, a CHQ-dehalogenase converted $\mathrm{CHQ}$ to $\mathrm{HQ}$ with release of chloride ion. The further degradation of HQ occurred via the ring-cleavage. The characteristics feature of this pathway is that both ammonium and chloride ions were released before the ring-cleavage. The potential of strain SPG to degrade 2C4AP in the soil was also investigated and observed that cells of strain SPG degraded 2C4AP efficiently in sterile as well as non-sterile soil. This strain is a good candidate for bioremediation of 2C4AP-contaminated sites. This is the first report of the 2C4AP degradation by any bacteria.

\section{Material and methods \\ Chemicals}

2C4AP, HQ and CHQ were purchased from SigmaAldrich. All other chemicals used were of high quality.

\section{Growth and degradation studies}

The overnight grown cells of strain SPG (1\%, v/v) were inoculated into $500 \mathrm{ml}$ Erlenmeyer flask containing $200 \mathrm{ml}$ minimal medium and $0.3 \mathrm{mM}$ 2C4AP as the sole source of carbon and energy. Samples collected at regular intervals $(0 \mathrm{~h}, 4 \mathrm{~h}, 8 \mathrm{~h}, 12 \mathrm{~h}, 16 \mathrm{~h}, 20 \mathrm{~h}, 24 \mathrm{~h}, 28 \mathrm{~h}$, $32 \mathrm{~h}, 36 \mathrm{~h}, 40 \mathrm{~h}, 44 \mathrm{~h}, 48 \mathrm{~h}, 52 \mathrm{~h}$ and $56 \mathrm{~h}$ ) were used for measuring the growth of cells of strain SPG, the 2C4AP degradation and analysis of chloride and ammonium ions. The growth of the cells of strain SPG was directly calculated by spectrophotometer at the absorbance of $600 \mathrm{~nm}$. For degradation studies, samples were centrifuged and extracted with ethyl acetate and the extracted samples were dissolved in the $20 \mu \mathrm{l}$ methanol and analyzed by high performance liquid chromatography using previously described method [1].

For the analysis of the chloride and ammonium ions, samples collected at regular intervals were centrifuged and supernatants were used to detect the chloride and ammonium ions. Ammonium ions were analyzed using Sigma Ammonia Assay kit. This kit is based on the reaction of the ammonia with $\alpha$-ketoglutaric acid in the presence of L-glutamate dehydrogenase to form L-glutamate. During this reaction, NADPH is oxidized to $\mathrm{NADP}^{+}$[20]. The ammonia concentration was measured by the decrease in absorbance at $340 \mathrm{~nm}$ because of the oxidation of NADPH [20]. The chloride ions were 
analyzed using QuantiChrom ${ }^{\mathrm{Tm}}$ Chloride assay kit (DICL250; BioAssay Systems, Hayward, CA, USA) [21]. The chloride ions present in the sample react with the mercuric 2,4,6-tripyridyl-s-triazine to form a colored complex. The color intensity of this compound was measured at $610 \mathrm{~nm}$ that is directly proportional to the chloride concentration in the sample [21].

\section{Identification of metabolites}

The overnight-grown cells of strain SPG (1\%,v/v) was inoculated into $1 \mathrm{~L}$ Erlenmeyer flask containing $500 \mathrm{ml}$ minimal medium and $0.3 \mathrm{mM} 2 \mathrm{C} 4 \mathrm{AP}$ as the sole source of carbon and energy. Samples $(50 \mathrm{ml})$ collected at regular intervals $(0 \mathrm{~h}, 12 \mathrm{~h}, 24 \mathrm{~h}, 36 \mathrm{~h}, 48 \mathrm{~h})$ were centrifuged and extracted with ethyl acetate. For extraction, the supernatant was mixed with equal volume of ethyl acetate in a separating funnel and shaked for 10-15 minutes [1]. After shaking, the ethyl acetate layer and aqueous layer were allowed to separate [1]. The ethyl acetate layer was collected and referred to as neutral extract [1]. The $\mathrm{pH}$ of the aqueous phase was then adjusted to 2.0 with $5 \mathrm{~N} \mathrm{HCl}$ and was re-extracted with equal volume of ethyl acetate [1]. After separation of two layers, the ethyl acetate layer was collected which was referred to as acidic extract and the aqueous layer was discarded [1]. The neutral and acidic extracts were mixed together and evaporated to dryness using an evaporator at $45^{\circ} \mathrm{C}$ [1]. The authentic standards (CHQ and HQ) were also extracted. For this, CHQ $(0.3 \mathrm{mM})$ or HQ $(0.3 \mathrm{mM})$ was added into $250 \mathrm{ml}$ Erlenmeyer flask containing $50 \mathrm{ml}$ minimal medium and sample was extracted as described above. The extracted samples were dissolved in $50 \mu \mathrm{l}$ methanol and analyzed using high performance liquid chromatography (HPLC) and gas chromatography-mass spectrometry (GC-MS).

HPLC analysis was performed using a Waters 600 model high performance liquid chromatography (HPLC) equipped with a photodiode array UV detector set at $300 \mathrm{~nm}$ [1]. The stationary phase was a $\mathrm{C}_{18}$ column and the injection volume was $15 \mu \mathrm{l}$. The mobile phase flow rate was $1.0 \mathrm{~mL} / \mathrm{min}$ and the mobile phase was composed of methanol (1\% glacial acetic acid) and water (1\% glacial acetic acid) at a ratio of 80:20.

GC-MS analysis was carried out using a GC-MSQP5000 instrument (Shimadzu, Tokyo, Japan) equipped with quadrupole mass filter [9]. The separation was performed using DB-1 capillary column with ionization of $70 \mathrm{eV}$. The column temperature was initially increased from $50^{\circ} \mathrm{C}$ to $190^{\circ} \mathrm{C}$ at the rate of $15^{\circ} \mathrm{C} / \mathrm{min}$ and then from $190^{\circ} \mathrm{C}$ to $280^{\circ} \mathrm{C}$ at the rate of $10^{\circ} \mathrm{C} / \mathrm{min}$. The carrier gas (nitrogen) flow rate was $15 \mathrm{ml} / \mathrm{min}$.

\section{Preparation of crude extracts and enzyme assays}

The cells of strain SPG were grown in $1 \mathrm{~L}$ Erlenmeyer flask containing $500 \mathrm{ml}$ minimal medium, $0.3 \mathrm{mM}$
2C4AP and $20 \mathrm{mM}$ sodium succinate. The $24 \mathrm{~h}$-grown cells of strain SPG were centrifuged and the pellet was washed with phosphate buffer $(50 \mathrm{mM}, \mathrm{pH} 7.5)$ and resuspended in the same buffer. The cells were sonicated and the cell extracts were centrifuged at $4^{\circ} \mathrm{C}$ for $15 \mathrm{~min}$ to remove cell debris [9]. The resulting supernatant was used for enzyme assays. The 2C4AP-deaminase activity was determined by measuring ammonia released from 2C4AP upon incubation with crude extract. The standard reaction mixture contained $50 \mathrm{mM}$ phosphate buffer ( $\mathrm{pH} 7.5), 0.2 \mathrm{mM} \mathrm{NADH}, 30 \mathrm{mg}$ protein (crude extract) and $300 \mu \mathrm{M} 2 \mathrm{C} 4 \mathrm{AP}$ in a total reaction volume of $2 \mathrm{ml}$. The reaction mixture was centrifuged upon incubation of $5 \mathrm{~min}$ and subjected for ammonia release and the GC-MS analysis as described above. The activities for CHQ-dehalogenase and the HQ dioxygenase were determined by the methods as described previously [9].

\section{Microcosm studies}

The experiments for microcosm studies were performed as described previously $[1,4,9]$. The composition of the soil used in this study was also similar as described previously [1]. The optimum conditions for degradation of 2C4AP were determined using the strain SPG and the chemical 2C4AP as described previously $[1,4,9,18]$. For microcosm studies, following four experiments with soil microcosms spiked with 100 ppm 2C4AP were performed (a) test microcosm with sterile soil in which cells of strain SPG were inoculated in the autoclaved soil, (b) test microcosm with non-sterile soil in which cells of strain SPG were inoculated in natural soil, (c) control microcosm with sterile soil where no bacterial strain is inoculated in the autoclaved soil, and (b) control microcosm with non-sterile soil where no bacterial strain is inoculated in natural soil. All soil microcosms were prepared in the $100 \mathrm{ml}$ beaker containing $20 \mathrm{~g}$ soil and covered with perforated aluminum foil. These microcosms were inoculated at $30^{\circ} \mathrm{C}$ for 10 days. The analysis of the 2C4AP degradation in soil was performed as described previously $[1,9,18,19]$. Samples were also analyzed by the GC-MS to identify the metabolites formed in the soil due to degradation of 2C4AP by strain SPG.

\section{Competing interests}

The authors declare that they have no competing interests.

\section{Authors' contributions}

PKA, AS, VS and HB conceived and designed the experiments, performed the experiments, analyzed the data, contributed reagents/materials/analysis tools, wrote the paper. TKM analyzed the data. All authors have read and approved the manuscript.

\section{Acknowledgment}

HB acknowledges "Cooperative Research Program for Agriculture Science \& Technology Development (PJ01049704)" Rural Development Administration, Republic of Korea for financial support. 
Received: 4 September 2014 Accepted: 14 November 2014

Published online: 27 November 2014

\section{References}

1. Arora PK, Srivastava A, Singh VP: Novel degradation pathway of 4-chloro-2-aminophenol via 4-chlorocatechol in Burkholderia sp. RKJ 800. Environ Sci Pollut Res Int 2014, 21:2298-2304.

2. Arora PK, Jain RK: Biotransformation of 4-chloro-2-nitrophenol into 5-chloro-2. methylbenzoxazole by a marine Bacillus sp. strain MW-1. Biodegradation 2012, 23:325-331.

3. Park H, Lim S, Chang YK, Livingston AG, Kim H: Degradation of chloronitrobenzenes by a coculture of Pseudomonas putida and a Rhodococcus sp. Appl Environ Microbiol 1999, 65:1083-1091.

4. Arora PK, Sharma A, Mehta R, Damodara B, Srivastava A, Singh VP: Metabolism of 4-chloro-2-nitrophenol in a gram-positive bacterium, Exiguobacterium sp. PMA. Microb Cell Factories 2012, 11:150.

5. Lendenmann U, Spain JC: 2-aminophenol 1,6-dioxygenase: a novel aromatic ring cleavage enzyme purified from Pseudomonas pseudoalcaligenes JS45. J Bacteriol 1996, 178:6227-6232.

6. Arora PK: Decolourization of 4-chloro-2-nitrophenol by a soil bacterium, Bacillus subtilis RKJ 700. PLoS One 2012, 7:e52012.

7. Arora PK: Metabolism of para-nitrophenol in Arthrobacter sp. SPG. E3 J Environ Sci Manag 2012, 3:52-57.

8. Arora PK, Srivastava A, Singh V: Novel degradation pathway of 2-chloro-4aminophenol in Arthrobacter sp. SPG. PeerJ PrePrints 2014, 2:e194v1.

9. Arora PK, Jain RK: Metabolism of 2-chloro-4-nitrophenol in a gram negative bacterium, Burkholderia sp. RKJ 800. PLoS One 2012, 7:e38676.

10. Arora PK, Sasikala C, Ramana VK: Degradation of chlorinated nitroaromatic compounds. Appl Microbiol Biotechnol 2012, 93:2265-2277.

11. Reddy GV, Gelpke MD, Gold MH: Degradation of 2,4,6-trichlorophenol by Phanerochaete chrysosporium: involvement of reductive dechlorination. J Bacteriol 1998, 180:5159-5164.

12. Miyauchi K, Adachi Y, Nagata Y, Takagi M: Cloning and sequencing of a novel meta-cleavage dioxygenase gene whose product is involved in degradation of gamma-hexachlorocyclohexane in Sphingomonas paucimobilis. J Bacteriol 1999, 181:6712-6719.

13. Miyauchi K, Suh SK, Nagata Y, Takagi M: Cloning and sequencing of a 2,5-dichlorohydroquinone reductive dehalogenase gene whose product is involved in degradation of gamma-hexachlorocyclohexane by Sphingomonas paucimobilis. J Bacteriol 1998, 180:1354-1359.

14. Arora PK, Srivastava A, Singh VP: Bacterial degradation of nitrophenols and their derivatives. J Hazard Mater 2014, 266:42-59.

15. Ju KS, Parales RE: Nitroaromatic compounds, from synthesis to biodegradation. Microbiol Mol Biol R 2010, 74:250-272.

16. Niu GL, Zhang JJ, Zhao S, Liu H, Boon N, Zhou NY: Bioaugmentation of a 4-chloronitrobenzene contaminated soil with Pseudomonas putida ZWL73. Environ Pollut 2009, 157:763-771.

17. Laha S, Petrova KP: Biodegradation of 4-nitrophenol by indigenous microbial populations in everglades soils. Biodegradation 1997, 8:349-356.

18. Ghosh A, Khurana M, Chauhan A, Takeo M, Chakraborti AK, Jain RK: Degradation of 4-nitrophenol, 2-chloro-4-nitrophenol, and 2,4-dinitrophenol by Rhodococcus imtechensis strain RKJ300. Environ Sci Tech 2010, 44:1069-1077.

19. Arora PK, Srivastava A, Singh VP: Degradation of 4-chloro-3-nitrophenol via a novel intermediate, 4-chlororesorcinol by Pseudomonas sp. JHN. Sci Rep 2014, 4:4475.

20. Ammonia Assay Kit [http://www.sigmaaldrich.com/content/dam/sigmaaldrich/docs/Sigma/Bulletin/aa0100bul.pdf]

21. QuantiChrom ${ }^{\text {TM }}$ Chloride Assay Kit [www.bioassaysys.com/file_dir/DICL.pdf]

doi:10.1186/s12934-014-0164-6

Cite this article as: Arora et al:: Metabolic pathway for degradation of 2-chloro-4-aminophenol by Arthrobacter sp. SPG. Microbial Cell Factories 2014 13:164.

\section{Submit your next manuscript to BioMed Central and take full advantage of:}

- Convenient online submission

- Thorough peer review

- No space constraints or color figure charges

- Immediate publication on acceptance

- Inclusion in PubMed, CAS, Scopus and Google Scholar

- Research which is freely available for redistribution 